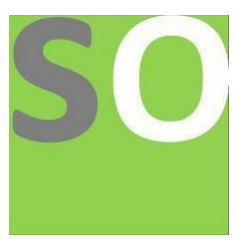

Article title: The Brocard conjecture

Authors: Jan Feliksiak[1]

Affiliations: N. A.[1]

Orcid ids: $0000-0002-9388-1470[1]$

Contact e-mail: jan.feliksiak1@yahoo.com

License information: This work has been published open access under Creative Commons Attribution License http://creativecommons.org/licenses/by/4.0/, which permits unrestricted use, distribution, and reproduction in any medium, provided the original work is properly cited. Conditions, terms of use and publishing policy can be found at https://www.scienceopen.com/.

Preprint statement: This article is a preprint and has not been peer-reviewed, under consideration and submitted to ScienceOpen Preprints for open peer review.

DOI: 10.14293/S2199-1006.1.SOR-.PPIH4LV.v1

Preprint first posted online: 21 February 2021

Keywords: Brocard conjecture, Maximal prime gaps bound, Prime Number Theorem, Twin primes, Least number of primes in Brocard's interval 


\title{
THE BROCARD CONJECTURE
}

\author{
JAN FELIKSIAK
}

Abstract.

The Brocard Conjecture asserts that the number of primes, within the interval, between the squares of two subsequent primes is:

$$
\left(\pi_{p_{(n+1)}^{2}}-\pi_{p_{(n)}^{2}}\right) \geq 4 \quad \forall p_{n} \in \mathbb{N} \mid n \geq 2
$$

Although the number of primes within this interval varies to a great degree, there is a common ground, which makes it possible to settle this old conundrum. Three bounds are developed: the least lower bound and the lower/upper bounds. The least lower bound is implemented to prove the conjecture. The lower/upper bounds exploit the shortest such interval, namely between the twin primes. This has been done in order to establish the bounds, on the smallest number of primes within that interval. The research objective was not only to provide a true/false answer, but to clarify some aspects of the distribution of prime numbers within this interval as well.

\section{(C)2020 Jan Feliksiak}

2000 Mathematics Subject Classification. 0102,1102, 1103, 11A41, 11K65, 11L20, 11N05.

Key words and phrases. Brocard Conjecture, maximal prime gaps upper bound, Prime Number Theorem, Twin Primes. 


\section{Preliminaries}

Within the scope of the paper, prime gap of the size $\mathfrak{g} \in \mathbb{N} \mid \mathfrak{g} \geq 2$ is defined as an interval between two primes $p_{n}, p_{(n+1)}$, containing $(\mathfrak{g}-1)$ composite integers. Maximal prime gap of the size $\mathfrak{g}$, is a gap strictly exceeding in size any preceding gap. All calculations and graphing were carried out with the aid of the Mathematica ${ }^{\circledR}$ software. For all $n \in \mathbb{N} \mid n \geq 8$ (unless indicated otherwise), we define:

Definition 1.1 (Maximal Prime Gap).

$$
\mathfrak{g}=p_{(n+1)}-p_{n}
$$

Theorem 1.2 (Maximal Prime Gaps Supremum and Infimum for primes).

For any $n \in \mathbb{N} \mid n \geq 11$ there exists at least one $p \in \mathbb{N} \mid n<p \leq n+c=t$; where $p$ is as usual a prime number and $c$ or upper bound on maximal prime gaps is given by:

$$
\begin{aligned}
\mathcal{U B}=c=\left\lfloor 5\left(\log _{10} n\right)^{2}-\frac{15}{8}\left(\log _{10} n\right)\right\rfloor \quad & \forall n \in \mathbb{N} \mid n \geq 11 \\
& \text { Equivalently, } p_{i+1}-p_{i} \leq c
\end{aligned}
$$

For the proof, please consult Feliksiak [8].

\section{Brocard Conjecture}

The Brocard's conjecture asserts that, there are at least four prime numbers between $p_{n}^{2}$ and $p_{(n+1)}^{2}$; where $p_{n}$ is as usual the $\mathrm{n}$-th prime number. Since the Brocard's assertion concerns successive squares of primes, we may begin with a general formula (please refer to a standard text for proofs) for the difference between the squares of two consecutive numbers. Let's denote two arbitrary integers $m, s \in \mathbb{N} \mid m=s+1$. Then the difference between their squares is given by:

$$
m^{2}-s^{2}=2 s+1
$$

For general integer squares $m, s \in \mathbb{N} \mid m>s$ we have to implement the sum to compute the difference:

$$
m^{2}-s^{2}=\sum_{k=s}^{m-1}(2 k+1)
$$

The sum 2.2 therefore, also produces the difference between two arbitrary squares of prime numbers for all $p_{n} \in \mathbb{N} \mid p_{n} \geq 2$.

Theorem 2.1 (Brocard Conjecture).

The Brocard conjecture holds for all $p_{n} \in \mathbb{N} \mid p_{n} \geq 3$ :

$$
\pi_{p_{(n+1)}^{2}}-\pi_{p_{(n)}^{2}} \geq 4
$$

Furthermore, the least number of primes within the interval increases as $p_{n}$ increases unboundedly. The least lower bound for the number of primes within that 
interval is given by:

$$
\mathcal{L L B}=\frac{4\left(p_{n}+1\right)}{\left(5\left(\log _{10} p_{n}^{2}\right)^{2}-\frac{15}{8}\left(\log _{10} p_{n}^{2}\right)\right)}
$$

Remark 2.1. The objective is to prove the least bound for primes within the interval $\left(p_{(n+1)}\right)^{2}-\left(p_{n}\right)^{2}$. The purpose of the least bound however, is solely to validate the Brocard conjecture. Obviously, at the twin primes we have the shortest such interval, necessarily therefore, the least number of primes will be found in that interval. Consequently, the whole proof of the Brocard Conjecture will proceed at the twin primes only, under the assumption that the twin primes continue indefinitely. This assumption will not in any way affect the validity of the proof, as it will be explained later on.

Proof.

From equation 2.2 in the case of twin primes, we obtain therefore for all $p_{n}, p_{(n+1)} \in \mathbb{N} \mid p_{n} \geq 3$ and $p_{(n+1)}=p_{n}+2$ :

$$
\left(p_{(n+1)}\right)^{2}-\left(p_{n}\right)^{2}=\sum_{k=p_{n}}^{\left(p_{(n+1)}-1\right)}(2 k+1)=2\left(p_{n}+p_{(n+1)}\right)=4\left(p_{n}+1\right)
$$

Theorem 1.2 states that there exists at least one prime number within $c$. Hence, the ratio $\left(p_{(n+1)}^{2}-p_{n}^{2}\right) / c$ measures the least multiplicity of prime numbers within that interval, which at the twin primes is given by:

$$
\frac{p_{(n+1)}^{2}-p_{n}^{2}}{\left(5\left(\log _{10} p_{n}^{2}\right)^{2}-\frac{15}{8}\left(\log _{10} p_{n}^{2}\right)\right)}=\frac{4\left(p_{n}+1\right)}{\left(5\left(\log _{10} p_{n}^{2}\right)^{2}-\frac{15}{8}\left(\log _{10} p_{n}^{2}\right)\right)}
$$

Evidently, increasing the difference between the respective primes e.g. in case of $p_{k}>p_{n}+2$, increases in direct proportion the value of ratio 2.6. Thus making the dependence, on the concept of infinitude of twin primes redundant. Suppose that for some $p_{n} \geq 59$ Theorem 2.1 is false. This implies that,

$$
\frac{4\left(p_{n}+1\right)}{\left(5\left(\log _{10} p_{n}^{2}\right)^{2}-\frac{15}{8}\left(\log _{10} p_{n}^{2}\right)\right)}<4
$$

However, at $p_{n}=59$, the inequality 2.7 attains $\sim 4.279780531$ and further increases. The limit of inequality 2.7 by the L'Hôpital's rule is:

$$
\lim _{n \rightarrow \infty}\left(\frac{4\left(p_{n}+1\right)}{\left(5\left(\log _{10} p_{n}^{2}\right)^{2}-\frac{15}{8}\left(\log _{10} p_{n}^{2}\right)\right)}\right)=\lim _{n \rightarrow \infty}\left(\frac{16(\log 10)^{2} p_{n}}{5\left(16 \log p_{n}^{2}-\log 1000\right)}\right) \rightarrow \infty
$$

Therefore, necessarily we have a contradiction to the initial hypothesis. The limit 2.8 , clearly indicates that the least number of primes within the interval increases, as $p_{n}$ increases unboundedly. Hence, for all $p_{n} \in \mathbb{N} \mid p_{n} \geq 59$, Theorem 2.1 holds. For all $p_{n} \in \mathbb{N} \mid 3 \leq p_{n} \leq 59$, direct evaluation evidently confirms that inequality 2.3, hence Theorem 2.1, hold in this range as well. Please refer to Fig. 1. Consequently, Theorem 2.1 holds $\forall p_{n} \in \mathbb{N} \mid p_{n} \geq 3$, concluding the proof. 

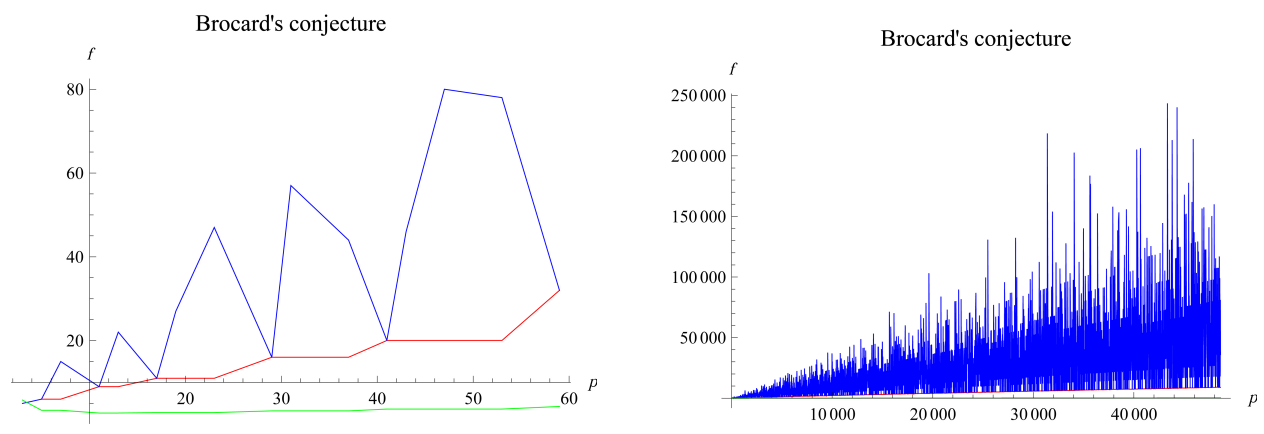

Figure 1. The drawings show the graphs of the output of the inequality 2.3. The L.H. figure shows the graph within the range $p_{n} \in \mathbb{N} \mid 3 \leq p_{n} \leq 59$. The R.H. figure shows the graph within the range $p_{n} \in \mathbb{N} \mid 3 \leq p_{n} \leq 48611$. The graph in Blue depicts the inequality 2.3 at every $n$ in the range. The Red curve depicts the inequality 2.3 at the twin primes only. The green curve shows the output of the R.H. side of the ratio 2.6.
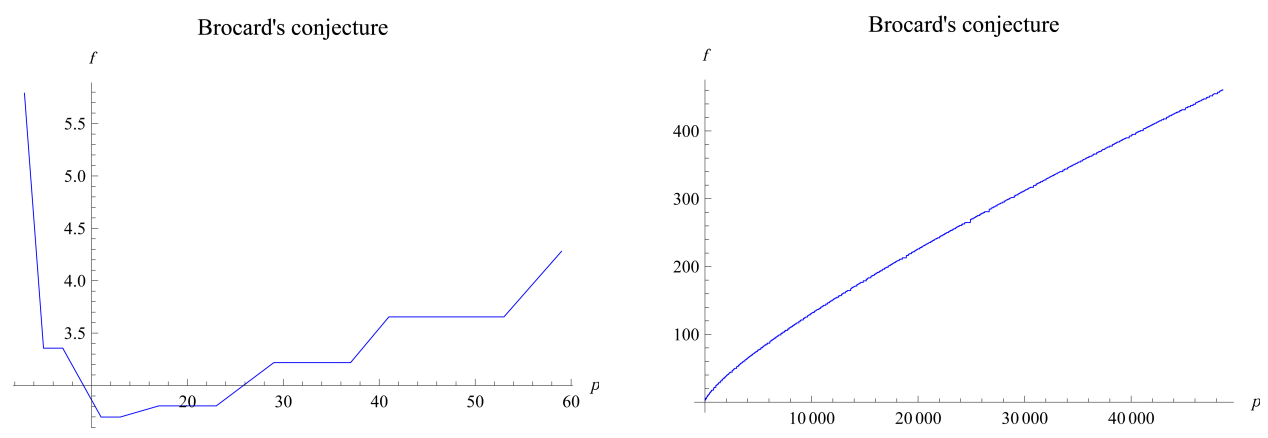

Figure 2. The drawings show the graphs of the output of the R.H. side of the ratio 2.6. The L.H. figure shows the graph within the range $p_{n} \in \mathbb{N} \mid 3 \leq p_{n} \leq 59$. The R.H. figure shows the graph within the range $p_{n} \in \mathbb{N} \mid 3 \leq p_{n} \leq 48611$. 
3. LOWER AND UPPER BOUNDS ON $\left(\pi_{p_{(n+1)}^{2}}-\pi_{p_{(n)}^{2}}\right)$

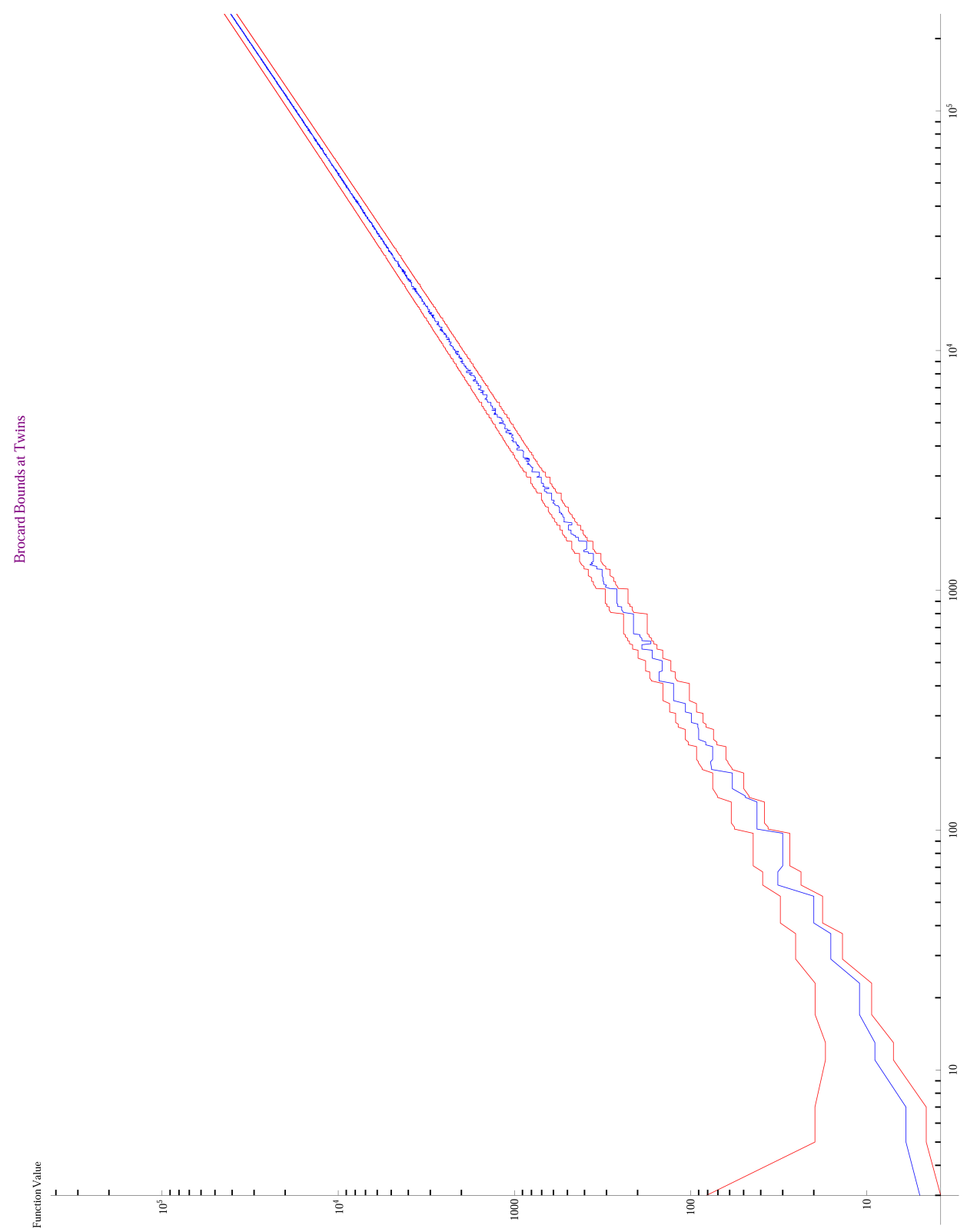

FiguRE 3. The log-log drawing shows the graphs of the difference $\left(\pi_{p_{(n+1)}^{2}}-\pi_{p_{(n)}^{2}}\right)$ (Blue) vs. the Lower and Upper Bounds (Red), at the twin primes, in the range $p_{n} \in \mathbb{N} \mid 3 \leq p_{n} \leq 2307229$. 
Theorem 3.1 (The Lower Bound On $\left(\pi_{p_{(n+1)}^{2}}-\pi_{p_{(n)}^{2}}\right)$ ).

The lower bound on the number of primes within the interval $p_{(n+1)}^{2}-p_{(n)}^{2}$, at the Twin Primes, for all $p_{n} \in \mathbb{N} \mid p_{(n)} \geq 3$ is given by:

$$
\mathcal{L B}=\left(\frac{2\left(p_{n}+1\right)}{\left(\log p_{n}\right)+1}\right)<\left(\pi_{p_{(n+1)}^{2}}-\pi_{p_{(n)}^{2}}\right)
$$

Proof.

Suppose that Theorem 3.1 is false for $p_{n} \in \mathbb{N} \mid p_{(n)} \geq 701$. This implies that in accordance with the hypothesis, at the twin primes we must have:

$$
\left(\pi_{p_{(n+1)}^{2}}-\pi_{p_{(n)}^{2}}\right)<\frac{2\left(p_{n}+1\right)}{\left(\log p_{n}\right)+1}
$$

The Cauchy's Root Test:

$$
\sqrt[n]{\left|a_{n}\right|}=\sqrt[n]{\left|\left(\pi_{p_{(n+1)}^{2}}-\pi_{\left.p_{(n)}^{2}\right)}\right)-\frac{2\left(p_{n}+1\right)}{\left(\log p_{n}\right)+1}\right|}
$$

at $p_{n}=701$ attains approximately 1.03002 and strictly from above, tends towards 1 as $p_{n}$ increases unboundedly. Please refer to Figure 4a. By the definition of the Cauchy's root test therefore, this implies that the sequence $a_{n}$ diverges. Consequently, in accordance with the hypothesis:

$$
\left(\pi_{p_{(n+1)}^{2}}-\pi_{p_{(n)}^{2}}\right)-\frac{2\left(p_{n}+1\right)}{\left(\log p_{n}\right)+1}<0
$$

the value of the inequality 3.4 must decrease, as $p_{n}$ increases unboundedly. However, at $p_{n}=701$, inequality 3.4 attains approximately 34.782 and further increases at a rate exceeding $k \sqrt{p_{n}}$ with $k=\log 2$, and the rate increases as $p_{n}$ increases unboundedly. Please refer to Figure 4b. Therefore, we have a contradiction to the initial hypothesis. Consequently, $\forall n \in \mathbb{N} \mid n \geq 701$ the inequality is valid:

$$
\left(\pi_{p_{(n+1)}^{2}}-\pi_{p_{(n)}^{2}}\right)>\frac{2\left(p_{n}+1\right)}{\left(\log p_{n}\right)+1}
$$

Direct computer evaluation for all $p_{n} \in \mathbb{N} \mid 3 \leq p_{(n)} \leq 701$ confirms that inequality 3.5 is valid in this range. Please refer to Figure 5. Consequently, we deduce that for all $p_{n} \in \mathbb{N} \mid p_{(n)} \geq 3$ Theorem 3.1 is valid as stated, concluding the proof.

Theorem 3.2 (The Upper Bound On $\left(\pi_{p_{(n+1)}^{2}}-\pi_{p_{(n)}^{2}}\right)$ ).

The upper bound on the number of primes within the interval $p_{(n+1)}^{2}-p_{(n)}^{2}$, at the Twin Primes, for all $p_{n} \in \mathbb{N} \mid p_{(n)} \geq 3$ is given by:

$$
\left(\pi_{p_{(n+1)}^{2}}-\pi_{p_{(n)}^{2}}\right)<\left(\frac{2\left(p_{n}+1\right)}{\left(\log p_{n}\right)-1}\right)=\mathcal{U B}
$$

Proof.

Suppose that Theorem 3.2 is false for $p_{n} \in \mathbb{N} \mid p_{(n)} \geq 701$. This implies that in accordance with the hypothesis, at the twin primes we must have:

$$
\left(\pi_{p_{(n+1)}^{2}}-\pi_{p_{(n)}^{2}}\right)>\frac{2\left(p_{n}+1\right)}{\left(\log p_{n}\right)-1}
$$



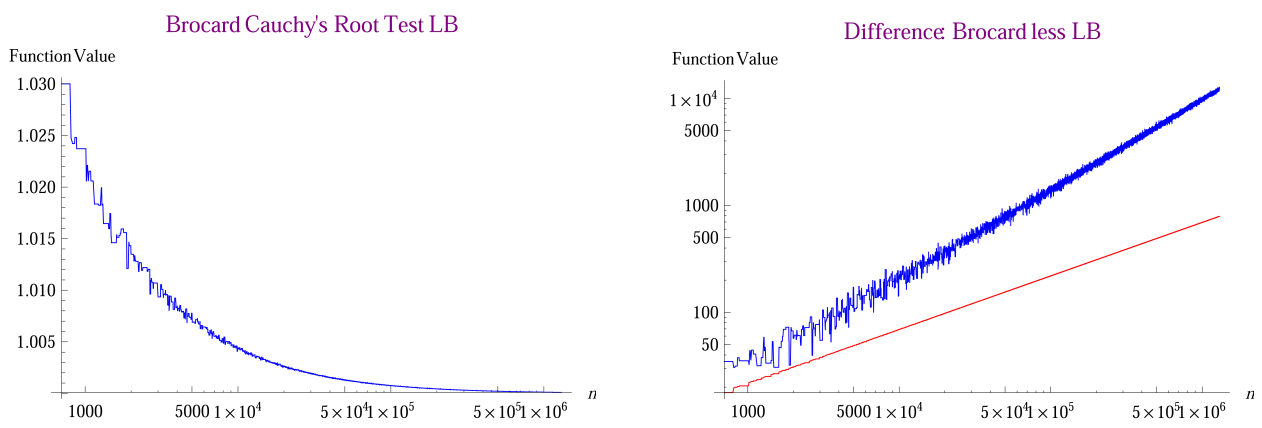

Figure 4. The L.H. figure shows the graph of the Cauchy Root Test of 3.3. The R.H. figure shows the graph of the difference 3.4 (Blue) vs $(\log 2) \sqrt{p_{n}}$ (Red). Both log-log figures are drawn in the range $p_{n} \in \mathbb{N} \mid 701 \leq p_{n} \leq 1299709$.

\section{Difference: Brocard less LB}

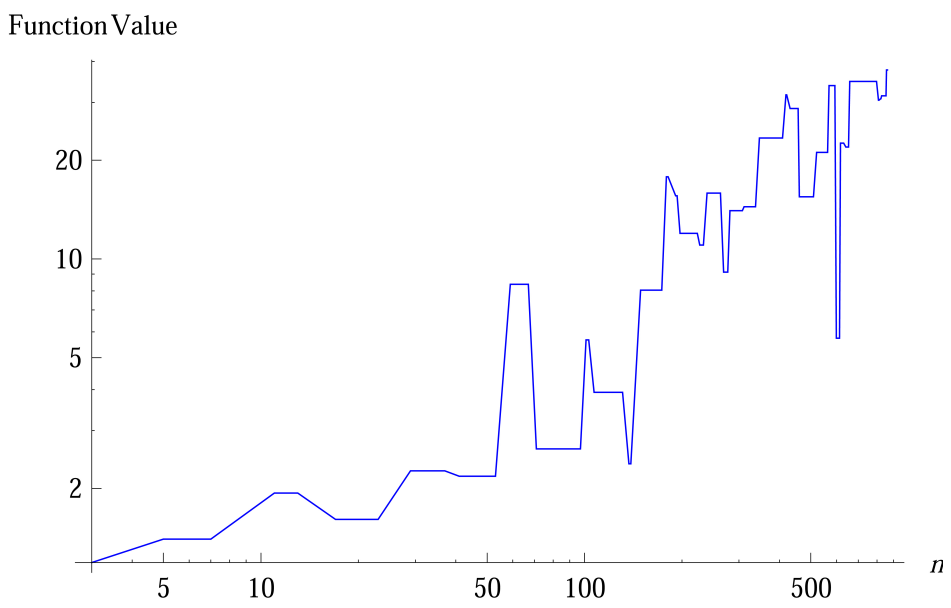

Figure 5. The figure shows the graph of the difference 3.4. The $\log$-log figure is drawn in the range $p_{n} \in \mathbb{N} \mid 3 \leq p_{n} \leq 863$.

The Cauchy's Root Test:

$$
\sqrt[n]{\left|a_{n}\right|}=\sqrt[n]{\mid \frac{2\left(p_{n}+1\right)}{\left(\log p_{n}\right)-1}-\left(\pi_{p_{(n+1)}^{2}}-\pi_{\left.p_{(n)}^{2}\right)}\right)}
$$

at $p_{n}=701$ attains approximately 1.02858 and strictly from above, tends towards 1 as $p_{n}$ increases unboundedly. Please refer to Figure 6a. By the definition of the Cauchy's root test therefore, this implies that the sequence $a_{n}$ diverges. Consequently, in accordance with the hypothesis:

$$
\frac{2\left(p_{n}+1\right)}{\left(\log p_{n}\right)-1}-\left(\pi_{p_{(n+1)}^{2}}-\pi_{p_{(n)}^{2}}\right)<0
$$

the value of the inequality 3.9 must decrease, as $p_{n}$ increases unboundedly. However, at $p_{n}=701$, inequality 3.9 attains approximately 29.4055 and further increases at 
a rate exceeding $k \sqrt{p_{n}}$ with $k=1$, and the rate rapidly increases as $p_{n}$ increases unboundedly. Please refer to Figure $6 \mathrm{~b}$. Therefore, we have a contradiction to the initial hypothesis. Consequently, $\forall n \in \mathbb{N} \mid n \geq 701$ the inequality is valid:

$$
\left(\pi_{p_{(n+1)}^{2}}-\pi_{p_{(n)}^{2}}\right)<\frac{2\left(p_{n}+1\right)}{\left(\log p_{n}\right)-1}
$$

Direct computer evaluation for all $p_{n} \in \mathbb{N} \mid 3 \leq p_{(n)} \leq 701$ confirms that inequality 3.10 is valid in this range. Please refer to Figure 7 . Consequently, we deduce that for all $p_{n} \in \mathbb{N} \mid p_{(n)} \geq 3$ Theorem 3.2 is valid as stated, concluding the proof.

Root Test Brocard Upper Bound

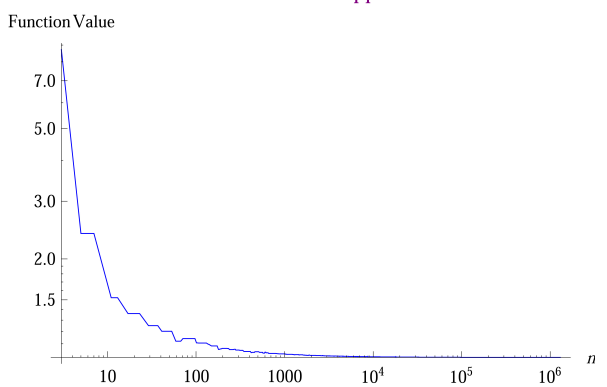

Difference: UB less Brocard

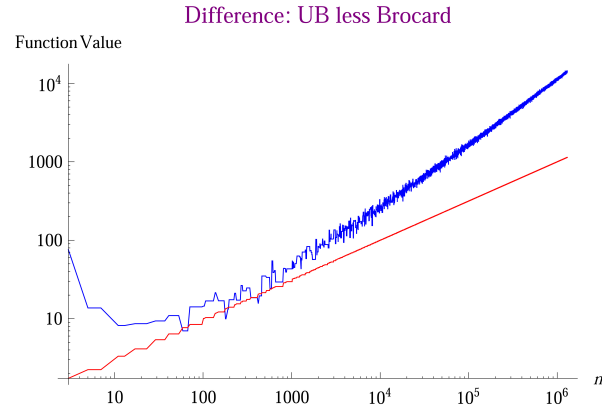

Figure 6. The L.H. figure shows the graph of the Cauchy Root Test of 3.8. The R.H. figure shows the graph of the difference 3.9 (Blue) vs $(\log 2) \sqrt{p_{n}}$ (Red). Both log-log figures are drawn in the range $p_{n} \in \mathbb{N} \mid 701 \leq p_{n} \leq 1299709$.

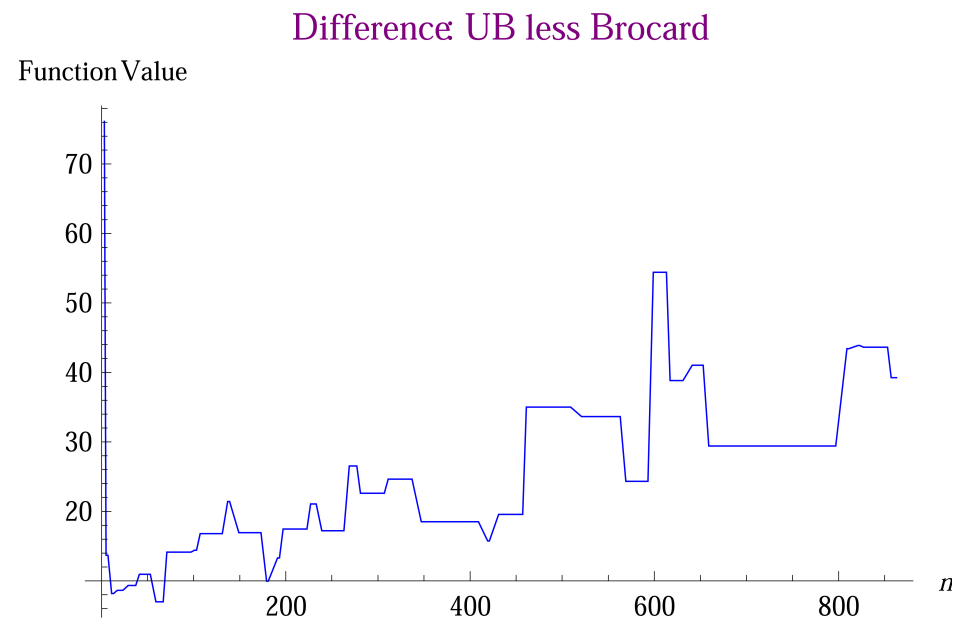

Figure 7 . The figure shows the graph of the difference 3.9. The $\log$-log figure is drawn in the range $p_{n} \in \mathbb{N} \mid 3 \leq p_{n} \leq 863$. 
Corollary 3.3 (Brocard Conjecture Summary.).

By Theorem 3.1 and Theorem 3.2, the difference $\left(\pi_{p_{(n+1)}^{2}}-\pi_{p_{(n)}^{2}}\right)$ at the Twin Primes, for all $p_{n} \in \mathbb{N} \mid p_{(n)} \geq 3$ has the following bounds:

$$
\mathcal{L B}=\frac{2\left(p_{n}+1\right)}{\left(\log p_{n}+1\right)}<\left(\pi_{p_{(n+1)}^{2}}-\pi_{p_{(n)}^{2}}\right)<\frac{2\left(p_{n}+1\right)}{\left(\log p_{n}-1\right)}=\mathcal{U B}
$$

With the average estimate $\mathcal{A} \mathcal{E}$, of the number of primes in the interval $\left(p_{(n+1)}^{2}-\right.$ $\left.p_{(n)}^{2}\right)$ :

$$
\left(\pi_{p_{(n+1)}^{2}}-\pi_{p_{(n)}^{2}}\right) \approx \frac{2\left(p_{n}+1\right)}{\log p_{n}}=\mathcal{A E}
$$

\section{Remark 3.1.}

The average estimate $\mathcal{A E}$ graph, considered at the Twin Primes, cuts through the graph of the difference $\left(\pi_{p_{(n+1)}^{2}}-\pi_{p_{(n)}^{2}}\right)$ in such a way that it persistently generates alternatively over/under estimates.

\section{REFERENCES}

[1] R.J. Backlund, Über die Differenzen zwischen den Zahlen, die zu den ersten $n$ Primzahlen teilerfremd sind. Commentationes in honorem Ernesti Leonardi Lindelöf, Annales Acad. Sci. Fenn. 32 (1929), no. 2, 1-9.

[2] A. Brauer and H. Zeitz, Über eine zahlentheoretische Behauptung von Legendre, Sitzungsbericht, Berliner Math. Ges. 29 (1930), 116-125.

[3] Chris K. Caldwell, The gaps between primes, 2006. http://primes.utm.edu/notes/gaps.html.

[4] Harald Cramer, On the order of magnitude of the difference between consecutive prime numbers, Acta Arithmetica (1936).

[5] Paul Erdös, On the difference of consecutive primes, Quarterly Journal Of Mathematics (1935).

[6] Paul Erdös and E.G. Strauss, Remarks on the differences between consecutive primes, Elem. Math. 35 (1980), 115-118.

[7] Jan Feliksiak, The elementary proof of the Riemann's Hypothesis, MDPI, AG, https://doi.org/10.20944/preprints202006.0365.v1 (2020).

[8] _ The maximal prime gaps Supremum and the Firoozbakht's Hypothesis No 30, MDPI, AG, https://doi.org/10.20944/preprints202006.0366.v1 (2020).

[9] — Maximal prime gaps bounds, ScienceOpen, https://doi.org/10.14293/S21991006.1.SOR-.PPWVKRR.v1 (2021).

[10] G.H. Hardy and E.M. Wright, An introduction to the theory of numbers, Oxford University Press, London, 1968

[11] D.R. Heath-Brown, Differences between consecutive primes, Jahresber. Deutsch. Mathem. Ver. 90 (1988), 71-89.

[12] Thomas R. Nicely, Some results of computational research in prime numbers (Computational number theory), 2009. http://www.trnicely.net.

[13] János Pintz, Very large gaps between consecutive primes, Journal Of Number Theory (1997).

[14] Robert A. Rankin, The difference between consecutive prime numbers, Journal London Mathematical Society (1938).

[15] Daniel Shanks, On maximal gaps between successive primes, Math. Comp. 18 (1964), 646651.

[16] Tomas Oliveira e Silva, Gaps between consecutive primes, 2006. www.ieeta.pt/ tos/gaps.html.

[17] K. Soundararajan, Small gaps between prime numbers: the work of Goldston-Pintz-Yildirim, Bulletin of the American Mathematical Society (2007).

[18] E. Westzynthius, Über die Differenzen Verteilung der Zahlen die zu den $n$ ersten Primzahlen teilerfremd sind, Comm. Phys. Math. Soc. Sci. Fenn. 5 (1931), 1-37. 